\title{
Estimation of free hemoglobin concentrations in blood bags by diffuse reflectance spectroscopy
}

\author{
Osman Melih Can \\ Yekta Ülgen
}




\title{
Estimation of free hemoglobin concentrations in blood bags by diffuse reflectance spectroscopy
}

\author{
Osman Melih Can ${ }^{a, \star}$ and Yekta Ülgen ${ }^{b}$ \\ ${ }^{a}$ Bogazici University, Institute of Biomedical Engineering, Istanbul, Turkey \\ ${ }^{\mathrm{b} B a h c e s e h i r}$ University, Faculty of Engineering and Natural Sciences, Department of Biomedical Engineering, Besiktas, Istanbul, Turkey
}

\begin{abstract}
Free hemoglobin (FHB) concentration is considered a prospect quality indicator for erythrocyte suspensions (ES) under storage. Storage lesions alter the optical properties of ES and can be monitored by diffuse reflectance spectroscopy. Due to storage lesions, erythrocytes lyse and release hemoglobin into the extracellular medium. The purpose of the study is to model and assess the quality of ES units in a blood bank with diffuse reflectance measurements together with hematological variables reflecting absorption and scattering characteristics of ES. FHB concentrations were modeled based on the increased scattering in the extracellular medium. A semiempirical model was used for relating optical properties of ES to the diffuse reflectance measurements. The attenuation in the blood bag was computed and its influence was discarded via normalization, in accordance with Monte Carlo simulations. In the experiments, 40 ES units were measured multiple times during prolonged storage of 70 days. A generalized linear model was used for modeling the training set, and, in the validation, the highest correlation coefficient between predicted and actual FHB concentrations was 0.89. Predicting the actual value was accurate at a maximum level of $R^{2}=0.80$. The error rate of the model in diagnosing the true quality was about 10\%. ( 2018 Society of Photo-Optical Instrumentation Engineers (SPIE) [DOI: 10.1117/1.JBO.23.12.127001]
\end{abstract} Keywords: blood; diffuse reflectance; free hemoglobin; scattering; optical properties; storage lesions; quality; blood bag. Paper 180407RR received Jun. 29, 2018; accepted for publication Nov. 21, 2018; published online Dec. 21, 2018.

\section{Introduction}

During storage, erythrocytes are exposed to unavoidable biochemical and morphological changes called storage lesions. ${ }^{1}$ The erythrocytes lyse and cellular hemoglobin is released into the cell suspending medium. The concentration of free hemoglobin (FHB) in the extracellular medium indicates the severity of the storage lesions. Regulatory agencies define the hemolysis level, which is calculated from FHB concentration, for measuring blood quality. Guidelines in Europe recommend maximum hemolysis level of $0.8 \%$ for blood products, and $1.0 \%$ is recommended in North America. ${ }^{2}$ However, in today's practice, quality control in national blood banks is carried out with the sampling method; some blood units are randomly selected from the population and their hemolysis levels are measured on the date of expiration. ${ }^{3}$ Since the test method is destructive, it is not applicable to all blood units before transfusion.

Currently, the only method for quality evaluation, prior to transfusion, involves physical examination of units and visual assessment of hemolysis. ${ }^{4,5}$ The redness of the suspending media is visually inspected for the presence of FHB, which turns the color of cell-free media from yellow to pink. In case of excessive FHB concentrations, the color becomes dark red or almost purple. Nevertheless, visual assessment of hemolysis level is subjective and mostly decisive. ${ }^{6}$

Rather than color perception, noninvasive spectrometric color measurement has been suggested. ${ }^{7}$ In that study, the color parameters derived from diffuse reflectance measurements were monitored during storage and found to correlate with hemolysis level. ${ }^{7}$ Results of that study showed that diffuse reflectance spectroscopy (DRS) may be used to assess the

*Address all correspondence to Osman Melih Can, E-mail: melih.can@boun .edu.t quality of stored blood; physiological changes during storage affect the optical properties of blood. DRS is also capable of analyzing optical properties of deeper layers of multilayered turbid samples such as blood under a thin plastic layer. ${ }^{8-11}$

In this paper, the diffuse reflectance spectra of erythrocyte suspensions (ES) were examined during storage. The purpose of this study is to model the effects of storage on the optical properties of ES for estimating the FHB concentration.

\section{Principles}

\subsection{Diffuse Reflectance Theory}

Light propagation in a turbid medium, such as ES, is generally expressed by the radiative transfer equation. ${ }^{12} \mathrm{~A}$ simpler exponential model was introduced by Zonios et al. for modeling diffuse reflectance from a homogeneous semi-infinite turbid medium. ${ }^{13}$ In the exponential model, the integral term of the radiative transfer equation was neglected and it was assumed that light is attenuated in the medium according to absorption and reduced scattering coefficients. Based on this model, a semiempirical equation was developed for relating measured diffuse reflectance $\left(R_{m}\right)$ with the optical properties: ${ }^{13}$

$R_{m}(\lambda)=c_{1}+\frac{\mu_{\mathrm{s}, \mathrm{ES}}^{\prime}(\lambda)}{c_{2} \times \mu_{\mathrm{a}, \mathrm{ES}}(\lambda)+c_{3}}$.

Here, $\mu_{\mathrm{a}, \mathrm{ES}}$ and $\mu_{\mathrm{s}, \mathrm{ES}}^{\prime}$ correspond to absorption and reduced scattering coefficients of ES, respectively, and $c_{1}, c_{2}$, and $c_{3}$ are the empirical parameters. The parameter $c_{1}$ was added to the semiempirical model in this study. It depends mostly on the optical properties of the blood bag. Other parameters $c_{2}$ and $c_{3}$ are

$1083-3668 / 2018 / \$ 25.00$ @ 2018 SPIE 
related to the measurement geometry in addition to the optical properties of the blood bag. This semiempirical expression [Eq. (1)] shows two fundamental characteristics of the reflectance: inverse dependence on the absorption coefficient and linear dependence on the reduced scattering coefficient. ${ }^{14}$ The reflectance model has been tested on many biological studies. $^{15-18}$

\subsection{Erythrocyte Suspensions and Their Optical Properties}

In the general preparation process of ES, blood is collected into a multibag system prefilled with an anticoagulant containing citrate, phosphate, and dextrose solution. The collected whole blood is centrifuged and then plasma is transferred into another bag. A 100-mL preserving solution containing saline, adenine, guanine, and mannitol (SAGM) is added to the remaining erythrocytes to replace plasma.

Erythrocytes are densely packed in ES; the hematocrit (HCT) of $\mathrm{ES}$ is around $55 \%$ to $70 \%$, while the lowest HCT of healthy women is $36 \%$ and the highest HCT of healthy men is $54 \%$. Consequently, concentration of red blood cells (RBCs) and hemoglobin are higher in ES than in the body. The count of $\mathrm{RBCs}$ in ES is around 5.5 to $7.0 \times 10^{12}(1 / \mathrm{L})$. With recent developments, the leukocytes are filtrated in ES and the number of residual white blood cells (WBCs) is decreased to $<10^{6} / \mathrm{L}$. In addition, the number of platelets (PLTs) is reduced, but their counts remain about $10^{10} / \mathrm{L}$. The normal range of single erythrocyte volume is about 80 to $100 \mu \mathrm{m}^{3}$, and cellular hemoglobin concentration is in the range of 320 and $360 \mathrm{~g} / \mathrm{L}$.

Optical properties of ES are very similar to those of whole blood, which have been extensively studied ${ }^{19-23}$ and reviewed ${ }^{24}$ ${ }^{26}$ in the literature. The absorption coefficient of ES is proportional to mean cell hemoglobin concentration (MCHC), ${ }^{27}$ and the forms of hemoglobin such as oxy- and deoxyhemoglobin characterize this absorption. The absorption coefficient of ES $\left(\mu_{\mathrm{a}, \mathrm{ES}}\right)$ is equal to

$$
\begin{aligned}
\mu_{\mathrm{a}, \mathrm{ES}}(\lambda)= & \mathrm{MCHC} \\
& \times\left\{\operatorname{Sat}\left(O_{2}\right) \varepsilon_{\mathrm{HbO}_{2}}(\lambda)+\left[1-\operatorname{Sat}\left(\mathrm{O}_{2}\right)\right] \varepsilon_{\mathrm{Hb}}(\lambda)\right\},
\end{aligned}
$$

where $\varepsilon_{\mathrm{HbO}_{2}}$ and $\varepsilon_{\mathrm{Hb}}$ are the molar extinction coefficients and $\operatorname{Sat}\left(\mathrm{O}_{2}\right)$ is the oxygen saturation level of hemoglobin.

Leukocytes, platelets, and FHB do not have much influence on the absorption properties of ES. ${ }^{22,28}$ Although FHB absorbs light, the light absorption of cellular hemoglobin is much higher since $\mathrm{MCHC}$ is at least 20 times denser than FHB concentration. It was also shown by Roggan et al. ${ }^{28}$ that FHB concentration has a very small effect on the absorption coefficient of whole blood. Therefore, MCHC sufficiently describes the absorption characteristics of whole blood.

The scattering properties of ES may be different from the scattering properties of whole blood since in ES the plasma is substituted with SAGM of which the refractive index is possibly different from the plasma.

The scattering properties are related to cell volumes and counts. ${ }^{29}$ Concentrations $\left(C_{n}\right)$, volumes $\left(V_{n}\right)$, and scattering cross-sections $\left(\sigma_{s, n}\right)$ of different cell types determine the scattering coefficient of ES $\left(\mu_{\mathrm{s}, \mathrm{ES}}^{\prime}\right)$ : $\mu_{\mathrm{s}, \mathrm{ES}}(\lambda)=\sum \frac{C_{n}}{V_{n}} \sigma_{s, n}(\lambda)$.

The contribution of different cells, RBCs, WBCs, and PLTs, on the scattering coefficient can be expressed as

$$
\mu_{\mathrm{s}, \mathrm{ES}}(\lambda)=\mu_{\mathrm{s}, \mathrm{RBC}}(\lambda)+\mu_{\mathrm{s}, \mathrm{WBC}}(\lambda)+\mu_{\mathrm{s}, \mathrm{PLT}}(\lambda)+\mu_{\mathrm{s}, \mathrm{EXT}}(\lambda)
$$

$\mu_{\mathrm{s}, \mathrm{EXT}}$ represents the light scattering coefficient, due to the particles in the extracellular medium. For example, scattering contributed by the platelets, $\mu_{\mathrm{s}, \mathrm{PLT}}$, would be proportional to the ratio of platelet concentrations in ES to mean platelet volume (MPV):

$\mu_{\mathrm{s}, \mathrm{PLT}} \propto \frac{\mathrm{PCT}}{\mathrm{MPV}}$,

where PCT is plateletcrit (\%). For leukocyte-depleted ES units, $\mu_{\mathrm{s}, \mathrm{WBC}}$ can be omitted. The reduced scattering coefficient of $\operatorname{RBCs}\left(\mu_{\mathrm{s}, \mathrm{RBC}}\right)$ is usually used to approximate the scattering coefficient of whole blood:

$\mu_{\mathrm{s}, \mathrm{ES}}(\lambda) \cong \mu_{\mathrm{s}, \mathrm{RBC}}(\lambda)=\gamma(\mathrm{HCT}) \frac{\mathrm{HCT}}{\mathrm{MCV}} \sigma_{\mathrm{s}, \mathrm{RBC}}(\lambda)$.

Here, MCV is mean cell volume of RBCs, $\sigma_{\mathrm{s}, \mathrm{RBC}}$ is the scattering cross section of RBCs, and $\gamma(\mathrm{HCT})$ is HCT-dependent correction factor. (1-HCT), $(1-\mathrm{HCT})^{2}$, and $(1-\mathrm{HCT})(1.4-$ HCT) were suggested by several authors for this empirical factor. $^{26,30-33}$

The reduced scattering coefficient of ES is proportional to the scattering coefficient but additionally depends on the anisotropy factor $g$ :

$\mu_{\mathrm{s}, \mathrm{ES}}^{\prime}(\lambda)=\mu_{\mathrm{s}, \mathrm{ES}}(\lambda)[1-g(\lambda)]$.

\subsection{Storage Lesions and Their Effects on Optical Properties}

During storage, erythrocytes undergo serious morphological and biochemical changes triggered by an inadequate adenosine triphosphate (ATP) production due to slowing down of glycolysis reactions in the refrigerated conditions. The lack of ATP inactivates the sodium-potassium pumps, and potassium ions leave RBCs, while sodium and calcium ions accumulate inside. The ionic exchanges between the intracellular and extracellular media alter their refractive indices and light scattering from cells at the interface between cell membrane and the extracellular space. Although glycolysis reactions slow down in low temperatures, RBCs continue to metabolize and the products of glycolysis make the media more acidic. The fall in $\mathrm{pH}$ reduces 2,3-diphosphoglycerate, which regulates binding of oxygen to hemoglobin and the hemoglobin oxygen affinity increases. ${ }^{34}$ The hemoglobin oxygenation process changes the absorption characteristics of the ES unit. Free radicals derived from oxygenation cause RBC injury by damaging membrane phospholipids and cytoskeletal proteins. These oxidative damages and the loss of intracellular water and ions result in crenation of erythrocytes. Subsequently, the erythrocytes swell and form a sphere shape with multiple long spicules. These shape changes would definitely affect the scattering crosssection of RBCs. Erythrocytes eventually loose these spicules 
as microvesicles into supernatant. ${ }^{35}$ Microvesicles carry cytoplasmic particles from their originated cells. The size of the microvesicles range from $100 \mathrm{~nm}$ to $1 \mu \mathrm{m}$, and their concentration progressively increases during storage. ${ }^{36}$ The accumulation of these micro-sized membrane particles in supernatant would increase the number of scattering events in the extracellular medium. The decreasing surface-to-volume ratio of cells with the membrane fragmentation decreases the deformability, and oxidative damages to structural components of RBCs make them susceptible to hemolysis. Erythrocytes finally rupture and release cytoplasmic particles and hemoglobin into the cell suspending medium. This leads to an increase of scattering in the extracellular medium of ES.

\subsection{Modeling of FHB Concentration}

Storage lesions modify both absorption and reduced scattering coefficient of ES as described in Sec. 2.3. However, FHB concentration, which is a substantial element for quality assessment of blood products, is expected to be in correlation with the increase of scattering from the extracellular medium. The combined effect of storage lesions on the overall reduced scattering coefficient of ES is unpredictable, but accumulation of microvesicles, cellular particles, and FHB into the extracellular medium would increase the contribution of scattering from the extracellular medium. For investigating the reduced scattering coefficient of extracellular medium $\left(\mu_{\mathrm{s}, \mathrm{EXT}}^{\prime}\right)$, Eq. (1) is rewritten as

$\mu_{\mathrm{s}, \mathrm{ES}}^{\prime}(\lambda)=c_{2}\left[R_{m}(\lambda)-c_{1}\right] \mu_{\mathrm{a}, \mathrm{ES}}(\lambda)+c_{3}\left[R_{m}(\lambda)-c_{1}\right]$.

After partitioning $\mu_{\mathrm{s}, \mathrm{ES}}^{\prime}$ as in Eq. (4) and omitting $\mu_{\mathrm{s}, \mathrm{WBC}}^{\prime}$, $\mu_{\mathrm{s}, \mathrm{EXT}}^{\prime}$ can be expressed as

$$
\begin{aligned}
\mu_{\mathrm{s}, \mathrm{EXT}}^{\prime}(\lambda)= & c_{2}\left[R_{m}(\lambda)-c_{1}\right] \mu_{a, \mathrm{ES}}(\lambda)+c_{3}\left[R_{m}(\lambda)-c_{1}\right] \\
& -\mu_{\mathrm{s}, \mathrm{RBC}}^{\prime}(\lambda)-\mu_{\mathrm{s}, \mathrm{PLT}}^{\prime}(\lambda) .
\end{aligned}
$$

Since the FHB concentration is associated with the increase in $\mu_{\mathrm{s} \text { EXT }}^{\prime}$, Eq. (9) can be used to model the FHB concentration. After substituting $\mu_{\mathrm{a}, \mathrm{ES}}, \mu_{\mathrm{s}, \mathrm{PLT}}^{\prime}$, and $\mu_{\mathrm{s}, \mathrm{RBC}}^{\prime}$ according to Eq. (2), Eqs. (5) and (6), respectively, the FHB concentration can be modeled with regressors depending on only diffuse reflectance and hematological parameters. Immeasurable covariates such as scattering cross-sections and anisotropy factor can be covered by the regression coefficients. The details and results of modeling are given in Secs. 3.4 and 4.5.

\section{Experimental Methods and Measurements}

\subsection{Measurement of Optical Properties of Blood Bag}

Optical properties of blood bags (Macopharma, Fr) were measured for investigating their contribution to diffuse reflectance data. Reflectance and transmittance of the blood bag were measured using two integrating spheres (Labsphere Inc.). In the measurement setup, an optical fiber, connected to the white light source (Ocean Optics, HL-2000), was directed to the entrance port of the first sphere and through the lens and diaphragm (Fig. 1). The incident light beam was focused on the blood bag, which was placed between the spheres. The reflected light and the transmitted light were integrated in first and second spheres, respectively. A fiber was connected

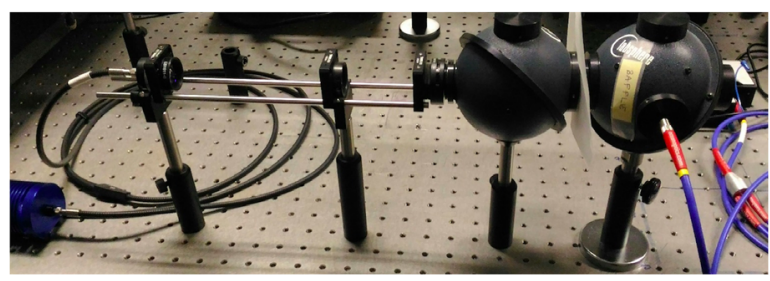

Fig. 1 Measurement setup of reflectance and transmittance of empty blood bag with two integrating spheres.

Table 1 The input parameters of the IAD method.

Incident beam diameter

$3.8 \mathrm{~mm}$

Thickness $(\times 2)$ of blood bag

$0.66 \mathrm{~mm}$

Diameter of spheres

$83.8 \mathrm{~mm}$

Diameter of the sample port of the spheres

$12.7 \mathrm{~mm}$

Diameter of the entrance port of the first sphere

$3.0 \mathrm{~mm}$

Diameter of the entrance port of the second sphere

$12.7 \mathrm{~mm}$

Diameter of the detector ports

$0.2 \mathrm{~mm}$

Wall reflectance

$99.0 \%$

to the detector ports of the spheres for transferring the light to the spectrometer (Ocean Optics, USB4000-VIS-NIR). During the measurements, air inside the bag was discharged with a vacuum pump because air may form an additional layer between two plastic sheets and produce errors in the measurements. The measured reflectance and transmittance were normalized with reference and dark measurements of both spheres. Finally, the inverse-adding-doubling (IAD) method ${ }^{37}$ was used to compute absorption and reduced scattering coefficients from the normalized reflectance and transmittance. The IAD program was run three times for different estimated anisotropy factors, $g=0.7,0.8$, and 0.9 . The refractive index of the blood bag was assumed to be 1.5. The IAD method requires additional inputs such as the dimensions of measurement geometry to calculate the optical properties. Input values are listed in Table 1.

\subsection{Monte Carlo Multilayer Simulations}

Effects of the optical properties of ES on the intensity of diffuse reflected light were simulated with the Monte Carlo multilayer code. ${ }^{38}$ Three layers were structured for the ES unit; the first and the last layers were the blood bag (thicknesses of $0.03 \mathrm{~cm}$ ). ES was in the mid-layer with a thickness of $1.54 \mathrm{~cm}$. The simulation was run 196 times, for 14 absorption coefficients of ES, logarithmically increasing from 1 to $3000 \mathrm{~cm}^{-1}$, and 14 scattering coefficients of ES ranged from 250 to $900 \mathrm{~cm}^{-1}$ in $50 \mathrm{~cm}^{-1}$ increments. The refractive index and anisotropy factor of ES were assumed to be 1.37 and 0.95 , respectively. The HenryGreenstein phase function was used for the random scattering process. $10^{6}$ photons were launched in each run, and the angle of incidence was set as $45 \mathrm{deg}$ to limit the optical depth. The total diffuse reflectance $\left(\mathrm{DR}_{\text {total }}\right)$ was considered for analysis. Changes in $\mathrm{DR}_{\text {total }}$, according to optical properties 
of ES, were recorded for testing the validity of the semiempirical model [Eq. (1)].

\subsection{Experiments}

This study was approved by the Ethics Committee of Institutional Review Board for Research with Human Subjects of Bogazici University, Istanbul, Turkey, and realized in cooperation with Turkish Red Crescent, Northern Marmara Regional Blood Center. Leukocyte-depleted 40 ES units supplied by the blood bank were used in the measurements. The ES units were stored at $4{ }^{\circ} \mathrm{C}$ in blood bank refrigerators for 70 days. Their diffuse reflectance, hematological parameters, and reference FHB concentrations were measured on the 1st, 42nd, 49th, 56th, 63rd, and 70th days of storage. These days were chosen for observing higher FHB concentrations. In statistical analysis, ES units were divided into two groups: 28 units were used for training and the remaining 12 units for validation.

\subsubsection{Diffuse reflectance measurements}

A reflection probe (Thorlabs, RP20) was used in diffuse reflectance measurements. The probe has six-around-one fibers with $0.2-\mathrm{mm}$ core diameters. The central fiber was used for transmitting the incident light from the optical source to the ES unit, and the fibers around the central one were used for transmitting the reflected light from the ES unit to the spectrometer. The spectrometer (Ocean Optics, USB4000-VIS-NIR) covers a wide range of the spectrum from 344 to $1038 \mathrm{~nm}$, and its optical resolution is about 1.5 to $2.3 \mathrm{~nm}$. The optical source was a tungsten halogen lamp (Ocean Optics, HL-2000). White diffuse reflectance standard (Ocean Optics, WS-1) was used to record the intensity of the incident light. Data acquisition was controlled by the software Oceanview (Ocean Optics Inc.).

Before starting each measurement, the optical source was warmed up for $20 \mathrm{~min}$. The ES unit was gently agitated to assess homogeneity inside the bag. Aggregated cells were separated, and the sedimented cells were mixed in this way. For the diffuse reflectance measurement, the ES unit was laid on a probe stand (Thorlabs, RPH-SMA). The probe holder was placed over the ES unit and tightened to an optical post. The probe was placed into the $45 \mathrm{deg}$ mount of the probe holder to avoid specular reflection. The setup was enclosed with black hardboard (Thorlabs, XE25C5D/M) to eliminate the interference of the ambient light on the measurements (Fig. 2).

The integration time for the spectrometer to count reflected photons was set to $5 \mathrm{~ms}$. The software was averaged over 100 scans before recording the data, and boxcar averaging width was adjusted to 25 for smoothing the collected data. After recording of the first measurement, the ES unit was removed and placed again under the probe and the measurements were repeated at least three times. The repeated measurements were averaged to minimize positioning and placement errors.

\subsubsection{Assessment of Sat $\left(\mathrm{O}_{2}\right)$ from diffuse reflectance spectrum of ES}

The hemoglobin oxygen saturation level $\left[\operatorname{Sat}\left(\mathrm{O}_{2}\right)\right]$ was estimated from diffuse reflectance measurements. Among the various techniques for quantification of oxyhemoglobin concentration from the reflectance spectra of the blood, ${ }^{39-42}$ the ratio of reflected light intensities at 760 and $790 \mathrm{~nm}$ was utilized to calculate blood oxygenation level: ${ }^{42}$

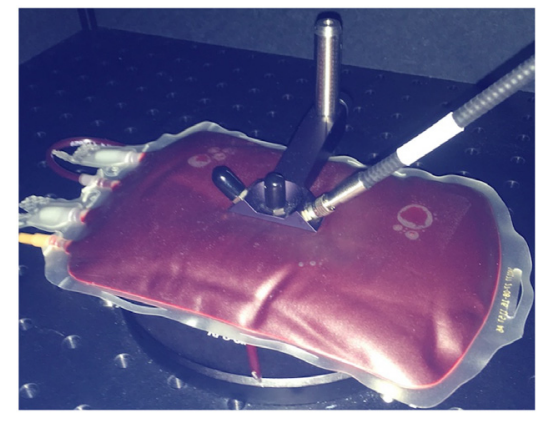

Fig. 2 Diffuse reflectance measurement of ES unit inside the dark box. The reflection probe was placed in the 45-deg aperture of the probe holder. The probe holder was tightened at the point where the probe connects the surface of the blood bag.

$\operatorname{Sat}\left(\mathrm{O}_{2}\right)(\%)=150.60 \frac{R_{m}(\lambda=760)}{R_{m}(\lambda=790)}-77.41$.

\subsubsection{Measurement of hematological parameters}

Following diffuse reflectance measurements, $5 \mathrm{~mL}$ of homogeneous blood was sampled from the bag. The sample was inserted under the probe of the hematology analyzer (Mindray, $\mathrm{BC} 3000)$ to measure the hematological variables: MCHC, HCT, $\mathrm{MCV}, \mathrm{PCT}$, and MPV.

\subsubsection{Reference FHB measurements}

Reference FHB concentrations of ES were measured from the sampled blood with the spectrometric Harboe technique. ${ }^{43}$ The sampled blood was centrifuged at $3000 \mathrm{rpm}$, for $20 \mathrm{~min}$, to obtain supernatant. The supernatant was diluted to $1: 10$, in $0.01 \% \mathrm{Na}_{2} \mathrm{CO}_{3}$ solutions and pipetted into a cuvette. Absorbance of supernatant relative to blank solution at $415 \mathrm{~nm}$, where hemoglobin has highest absorption peak, was read by a bench-top spectrometer (Beckman Coulter DU 730). Additionally, absorbance $(A)$ at 380 and $450 \mathrm{~nm}$ was also read to extract interferences of the proteins and lipids, respectively. Finally, the following expression yields the FHB concentration: ${ }^{44}$

$$
\begin{aligned}
\operatorname{FHB}(\mathrm{g} / \mathrm{L})= & 0.836 \\
& \times[2 \times A(\lambda=415)-A(\lambda=380)-A(\lambda=450)] .
\end{aligned}
$$

\subsection{Generalized Linear Model for Predicting FHB Concentration}

GLM was used to relate the FHB concentration with the reduced scattering coefficient of extracellular medium. The general expression of GLM is

$\mathbf{X} \cdot \mathbf{b}+\mathbf{e}=\mathbf{Y}$

where $\mathbf{X}$ is the design matrix and $\mathbf{b}, \mathbf{e}$, and $\mathbf{Y}$ are the vectors of regression coefficients, residuals, and outcome variable, respectively. The design matrix is composed of the predictors $\left(X_{p}\right)$. Diffuse reflectance and hematological variables were used as predictors, and they were formatted according to Eq. (9), which expressed $\mu_{\mathrm{s}, \mathrm{EXT}}^{\prime} \cdot \mu_{\mathrm{s}, \mathrm{EXT}}^{\prime}$ is replaced with FHB concentration as the outcome variable of the model. At a selected wavelength $\lambda$, the matrix representation of GLM is 


$$
\left[\begin{array}{cccc}
X_{1}^{1,1} & X_{2}^{1,1} & \cdots & X_{p}^{1,1} \\
\vdots & \vdots & & \vdots \\
X_{1}^{1, k} & X_{2}^{1, k} & & X_{p}^{1, k} \\
X_{1}^{2,1} & X_{2}^{2,1} & & X_{p}^{2,1} \\
\vdots & \vdots & & \vdots \\
\vdots & \vdots & & \vdots \\
X_{1}^{n, k} & X_{2}^{n, k} & \ldots & X_{p}^{n, k}
\end{array}\right]_{(n \times k) \times p}^{\lambda} \quad\left[\begin{array}{c}
b_{1} \\
b_{2} \\
\vdots \\
b_{p}
\end{array}\right]_{p \times 1}^{\lambda}+\left[\begin{array}{c}
e_{1,1} \\
\vdots \\
e_{1, k} \\
e_{2,1} \\
\vdots \\
\vdots \\
e_{n, k}
\end{array}\right]_{(n \times k) \times 1}=\left[\begin{array}{c}
Y_{1,1} \\
\vdots \\
Y_{1, k} \\
Y_{2,1} \\
\vdots \\
\vdots \\
Y_{n, k}
\end{array}\right]_{(n \times k) \times 1}
$$

Indices $p, n$, and $k$ represent the number of predictors, the number of ES units, and the number of repeated measurements during storage, respectively. GLM was applied to $28 \mathrm{ES}$ units in the training set for all wavelengths from 344 to $1000 \mathrm{~nm}$ with 2$\mathrm{nm}$ increments. The goodness of fits, RMS errors, and statistical significance of the regression coefficients were analyzed, and the models were tested on 12 ES units in the validation set. Matlab 2017b (Mathworks Inc.) was used for statistical analysis; the results are given in Secs. 4.5 and 4.6.

(a)

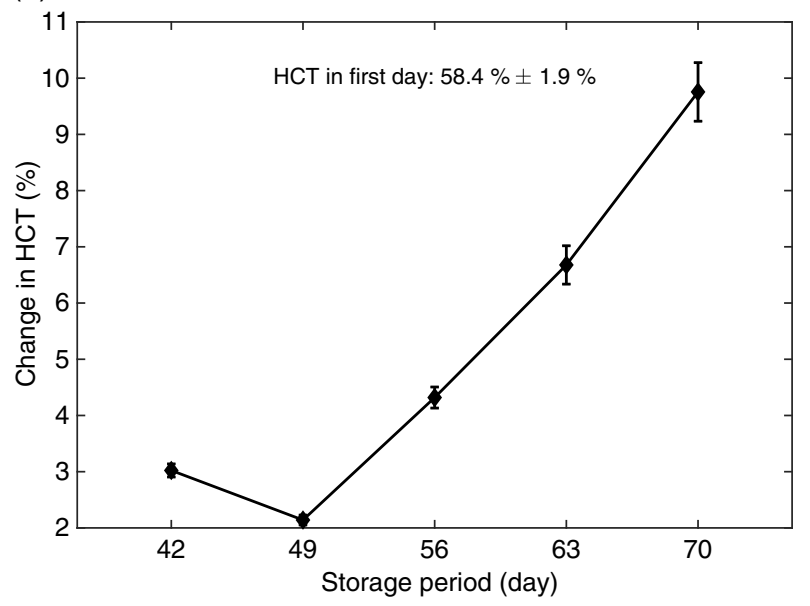

\section{Results}

\subsection{Storage Effects on Hematology of ES}

During the 70 days of storage, ES units underwent storage lesions and a gradual increase in FHB concentration was observed. The standard deviations between the FHB concentrations of 40 samples also increased with the storage period. The critical level of FHB concentration in blood bags is about 3 to

(b)

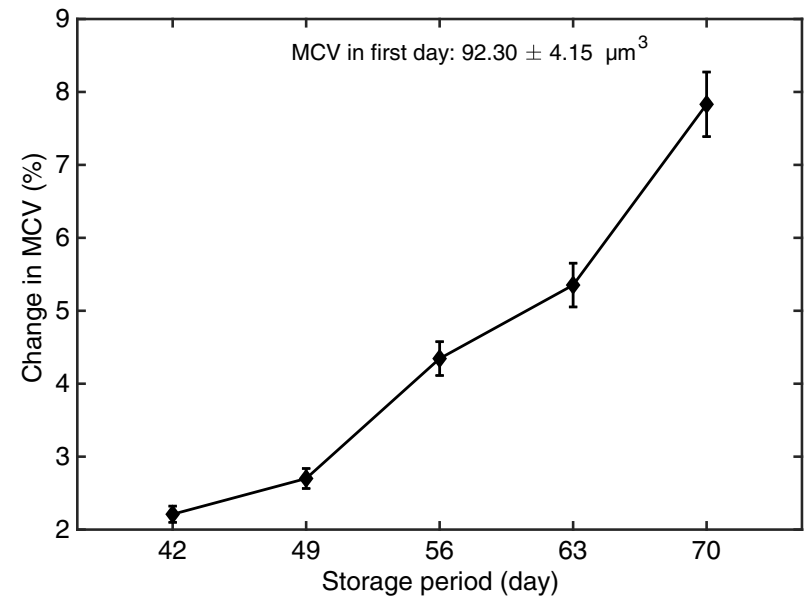

(c)

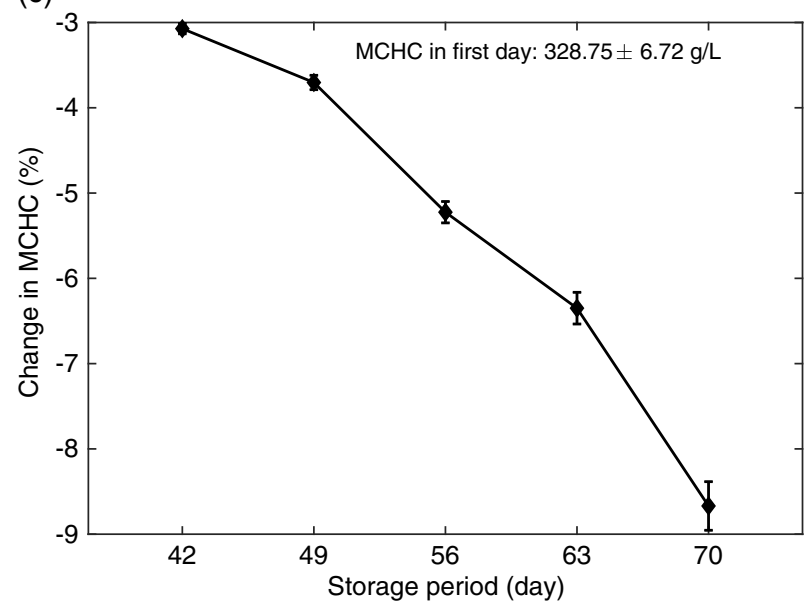

Fig. 3 Effect of storage on (a) HCT, (b) MCV, and (c) MCHC, measured with the hematology analyzer after sampling blood from ES units. 
$6.5 \mathrm{~g} / \mathrm{L}$. FHB concentration of all ES units was measured below the critical level for the first 42 days, but it became critical on the 56th day of storage and thereafter. On the 70th day, almost all ES units were beyond the acceptable quality limits. Hematological parameters that directly affecting the optical properties have also changed significantly with prolonged storage. An increase in HCT and MCV was observed while there was a fall in MCHC (Fig. 3). Repeated measures-ANOVA results showed that storage had a significant effect on these hematological variables $(p<0.001)$. Changes in PCT and MPV were not statistically significant. The correlation coefficient between FHB concentration and MCHC, HCT, and MCV were $0.734,0.6863$, and 0.5524 , respectively.

\subsection{Diffuse Reflectance Spectra of ES Units and Effects of Storage on Them}

The diffuse reflectance spectrum of an arbitrarily chosen ES unit is plotted in Fig. 4, for the first day of storage. The diffuse reflectance spectrum exhibited the absorption characteristics of hemoglobin dominantly. The inverse relationship between absorption and measured diffuse reflectance, as in Eq. (1), can be seen in Fig. 4. A valley in a particular region of diffuse reflectance spectrum corresponded to the peak molar extinction coefficient of hemoglobin at that wavelength. However, this inverse proportionality was broken in the 400 - to 450 -nm region

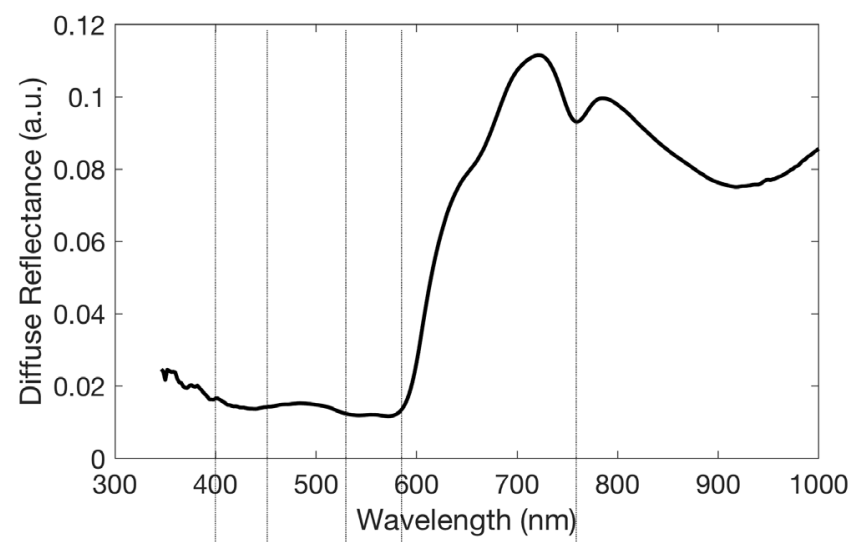

(a)

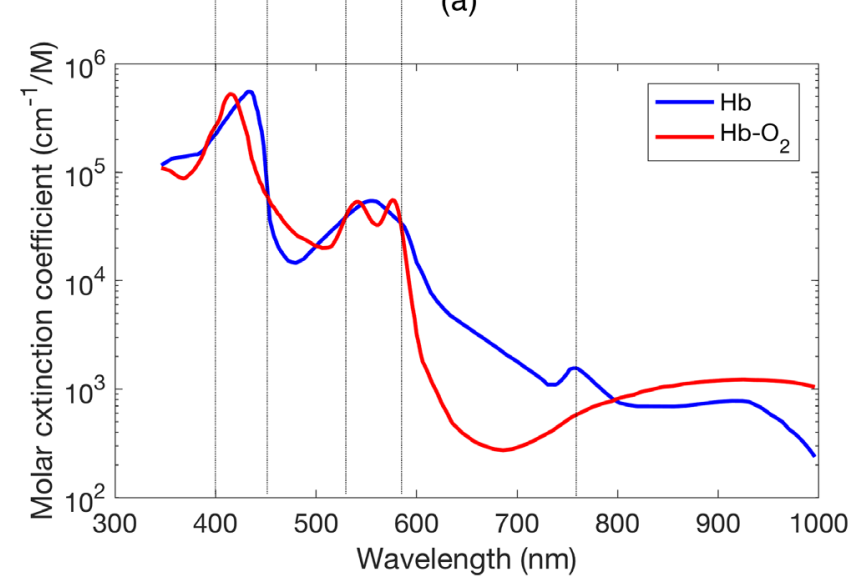

(b)

Fig. 4 (a) The diffuse reflectance of an ES unit, measured on the first day and (b) oxy- and deoxyhemoglobin absorption curves (data are from Ref. 45) are shown.

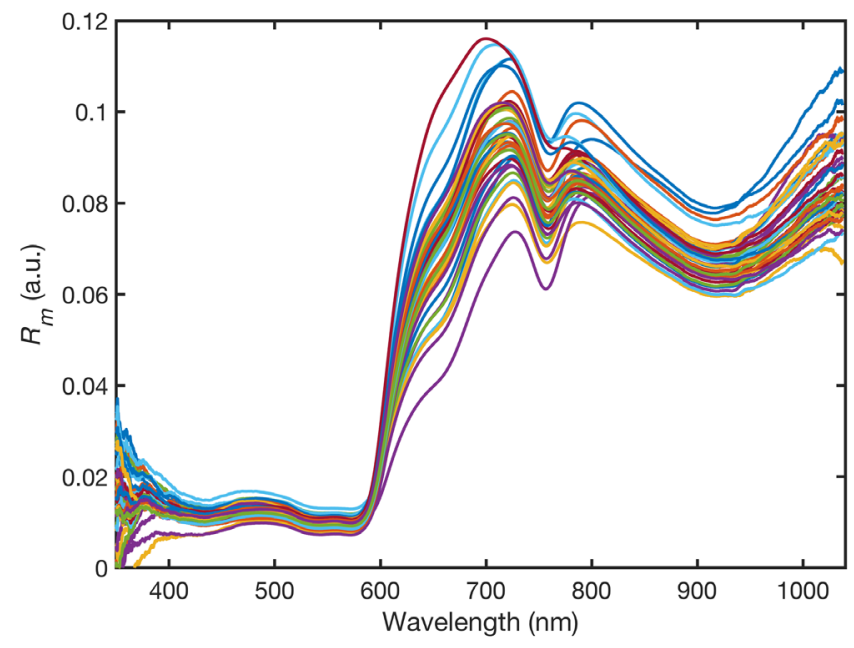

Fig. 5 Diffuse reflectance spectra of $40 \mathrm{ES}$, on the first day of storage.

[Fig. 4(b)], where the absorption of hemoglobin molecules is highest, but it was not the region having lowest reflected light intensity, in diffuse reflectance spectra [Fig. 4(a)]. The diffuse reflectance spectrum had a constant base level in the region where the absorption of ES was higher. In this region, the detected light mostly contained the reflections from the blood bag.

The first day measurements of the diffuse reflectance spectra of ES units showed large deviation among them (Fig. 5). It is likely that variations in their hematological parameters caused such difference in their optical properties, i.e., in their diffuse reflectance spectra.

With storage time, it was observed that diffuse reflectance of ES units generally increased between 600 and $750 \mathrm{~nm}$ and decreased beyond $800 \mathrm{~nm}$. The peak wavelength of the spectrum shifted to the left. The two peaks around 725 and $790 \mathrm{~nm}$ turned into a single peak around 670 to $680 \mathrm{~nm}$, and the valley around $760 \mathrm{~nm}$ diminished in some of the ES units. These changes were associated with the changes in the hemoglobin oxygenation level, calculated with Eq. (10). The oxygenation levels of ES units increased with storage time (Fig. 6). The direction of the shift in diffuse reflectance spectra due to increasing

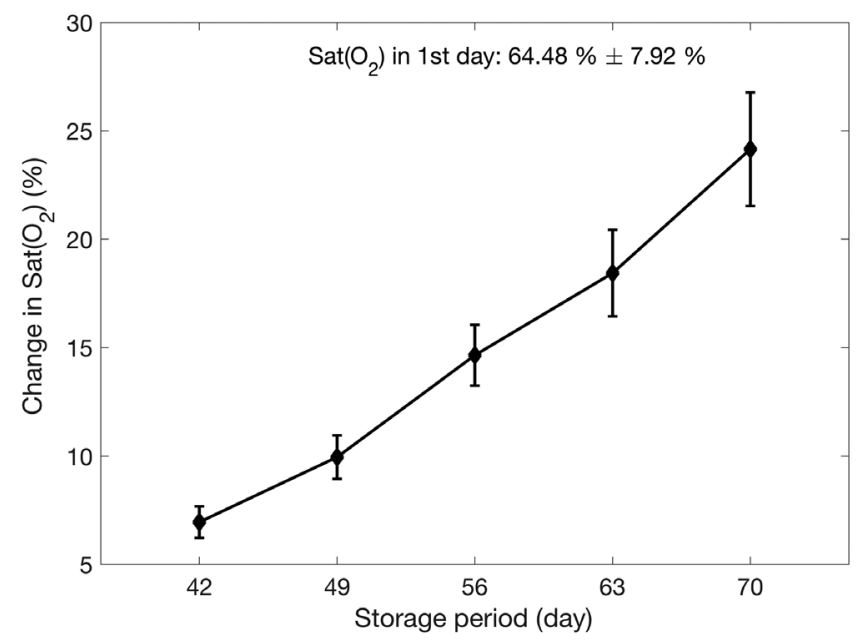

Fig. 6 Percent change of oxygenation during storage. 


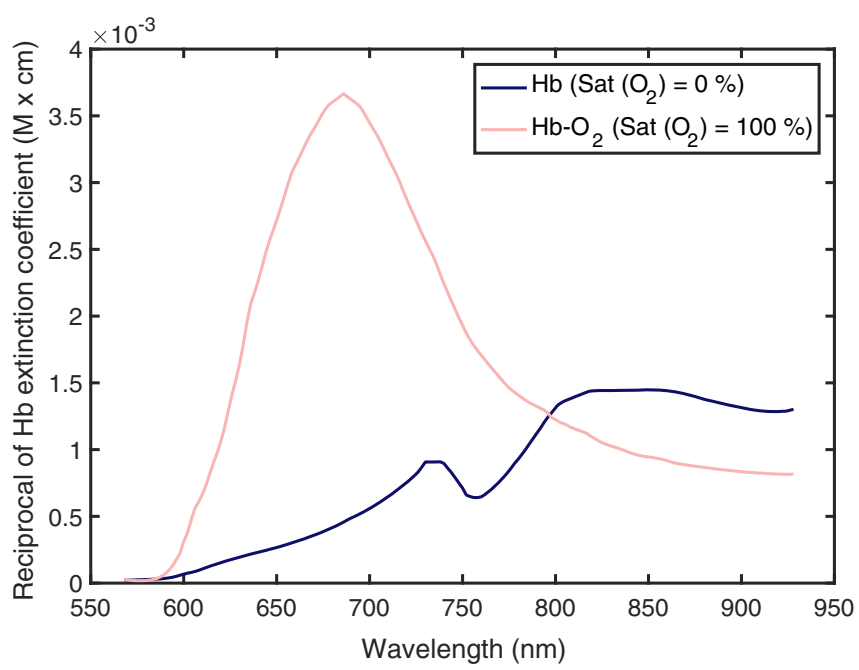

(a)

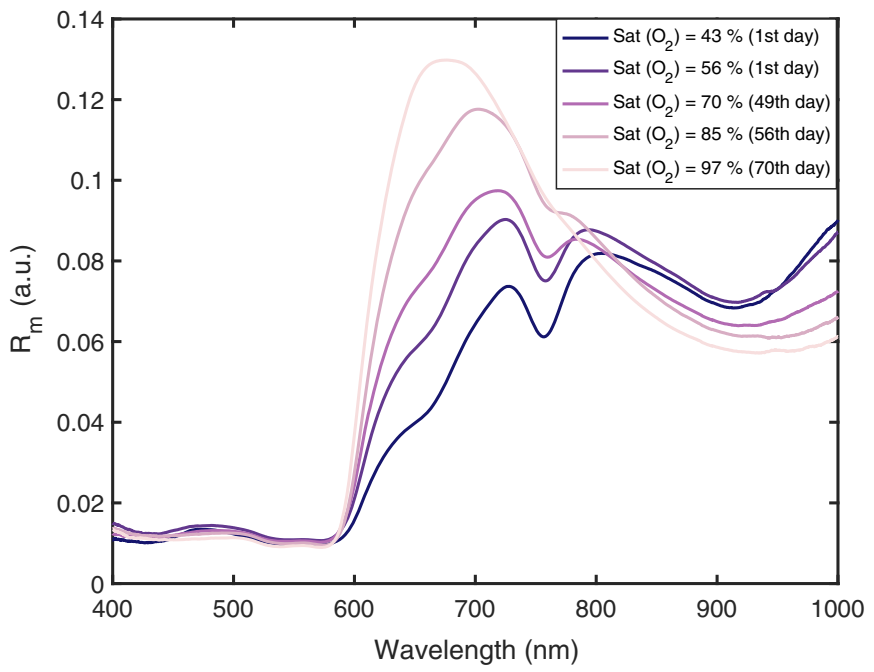

(b)

Fig. 7 Shifts in diffuse reflectance spectra with increasing oxygenation and its correlation with the reciprocal of molar extinction coefficient of hemoglobin. (a) Reciprocal of the molar extinction coefficient of reduced and fully oxygenated hemoglobin, ${ }^{45}$ (b) the diffuse reflectance spectra of different ES units measured at different storage times.

oxygenation [Fig. 7(b)] could be estimated from the reciprocal of hemoglobin molar extinction spectra [Fig. 7(a)].

There was no strong correlation between the changes at any wavelength of the diffuse reflectance spectra and FHB concentration. The most powerful correlation was observed in the range of 800 to $1000 \mathrm{~nm}$, and it was a negative correlation of around 0.6.

\subsection{Optical Properties of Blood Bag}

The absorption and reduced scattering coefficients of the blood bag were computed with the IAD method. Between 550 and $900 \mathrm{~nm}$, the absorption coefficient of the blood bag was between 0.5 and $0.7 \mathrm{~cm}^{-1}$, and the reduced scattering coefficient was around $7 \mathrm{~cm}^{-1}$ (Fig. 8). Beyond this region, higher fluctuations were observed as the power of the halogen source decreased and the measurement signal-to-noise ratio became poor. The blood bags were semitransparent; the inner surface was generally modified to provide an appropriate contact with erythrocytes and minimize hemolysis. These textures lead to high scattering and absorption coefficients. ${ }^{46}$ Even higher scattering coefficients were presumed in Ref. 47 . The anisotropy factor had very little influence on the computations of the reduced scattering and absorption coefficients.

\subsection{Validity of Exponential Model}

The measured optical properties of the blood bag were used as inputs to Monte Carlo multilayer (MCML) simulations to investigate the semiempirical reflectance model [Eq. (1)]. The absorption coefficient of the blood bag was set to $0.6 \mathrm{~cm}^{-1}$ and the scattering coefficient of the blood bag was calculated as $35 \mathrm{~cm}^{-1}$ while the anisotropy factor was selected as 0.8 and the reduced scattering coefficient of the blood bag was set to $7 \mathrm{~cm}^{-1}$.

The simulated data were in a good agreement with Eq. (1), when the data were divided into two parts according to absorption levels of ES. The goodness of fits were 0.9287 and 0.992 , respectively, for low and high absorption levels (Fig. 9). The model seemed to be slightly better when $\mu_{\mathrm{a}, \mathrm{ES}}$ was higher. Nevertheless, the fitness of the model for low $\mu_{\mathrm{a}, \mathrm{ES}}$ was also acceptable. At low absorption levels of ES $\left(\mu_{\mathrm{a}, \mathrm{ES}} \leq 5 \mathrm{~cm}^{-1}\right)$, the relationship between the reduced scattering coefficient and diffuse reflectance became nonlinear. At very high absorption levels of ES, however, total diffuse reflectance converged to $c_{1}$ as in Eq. (1).

The validity of Eq. (1) was also tested with the experimental data of the training set. Initially, diffuse reflectance at $570 \mathrm{~nm}$ was used for normalization, where the intensity of measured reflectance was minimum, to eliminate the empirical coefficient $c_{1}$, which is asymptotically equal to the measured diffuse reflectance at higher absorption levels of ES. The normalized diffuse reflectance spectra $R_{\text {norm }}(\lambda)$ was acquired by subtracting the reflectance at $570 \mathrm{~nm}$ from the spectra:

$R_{\text {norm }}(\lambda)=R_{m}(\lambda)-R_{m}(\lambda=570)$.

Following normalization, the optical properties $\mu_{\mathrm{a}, \mathrm{ES}}$ and $\mu_{\mathrm{S} \text {.ES }}^{\prime}$ in Eq. (1) were replaced with the hematological parameters MCHC, HCT, MCV, and Sat $\left(\mathrm{O}_{2}\right)$ according to Eqs. (2) and (6), respectively. Then, with these hematological parameters, the normalized reflectance values were predicted according to Eq. (1). The predicted values were in good agreement with the actual normalized diffuse reflectance values (Fig. 10). The best goodness of fit was 0.8922 , obtained at 658 and $660 \mathrm{~nm}$. Nonetheless, the goodness of fits were greater than 0.8 in all wavelengths from 590 to $700 \mathrm{~nm}$.

\subsection{Results of GLM for FHB Predictions}

For estimating the response variable FHB concentration, the selected predictors of GLM were 


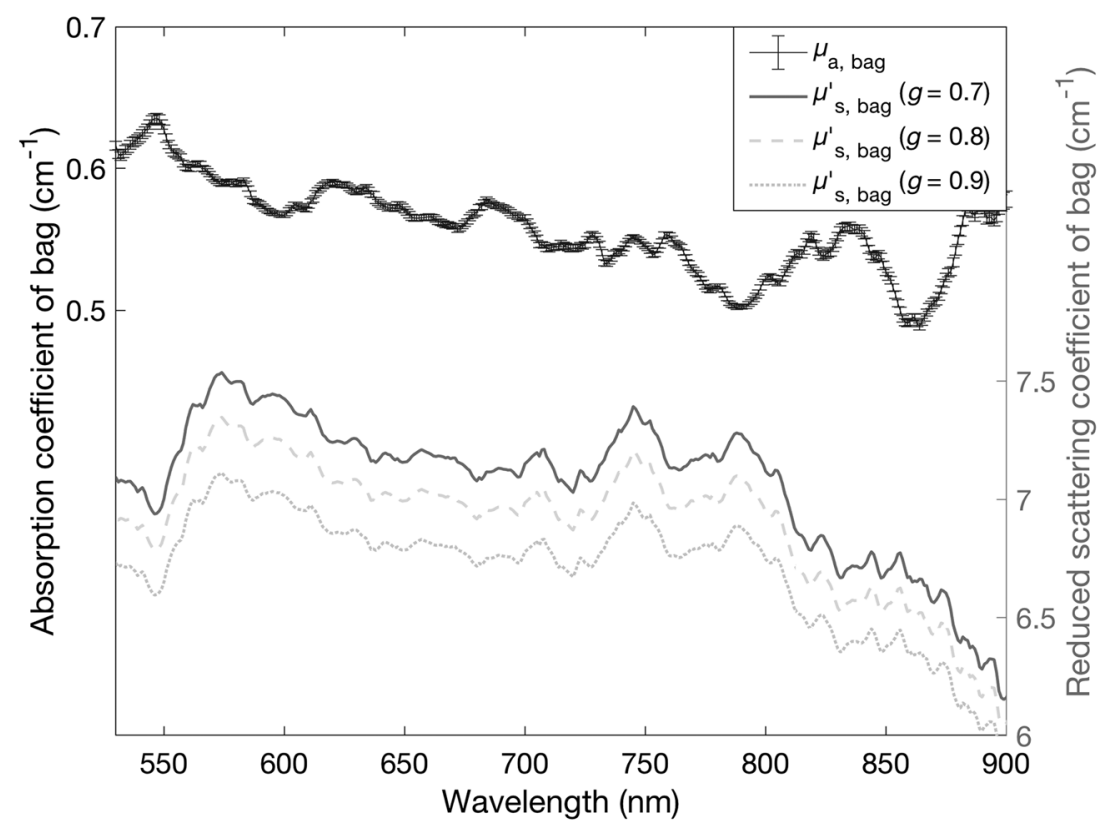

Fig. 8 Spectra of absorption (left axis) and reduced scattering coefficients (right axis) of the blood bag. Actual measurements were smoothed for both spectra. The error bars in the absorption spectra represent the standard deviations of measurements with different anisotropy factors $(g=0.7,0.8$, and 0.9$)$.

$$
\begin{aligned}
X_{1} & =1, \\
X_{2}(\lambda) & =R_{\text {norm }}(\lambda) \times \frac{1}{1-\mathrm{HCT}}, \\
X_{3}(\lambda) & =R_{\text {norm }}(\lambda) \times \mathrm{MCHC} \times \operatorname{Sat}\left(\mathrm{O}_{2}\right) \times \varepsilon_{\mathrm{Hb}-\mathrm{O}_{2}}(\lambda) \times \frac{1}{1-\mathrm{HCT}}, \\
X_{4}(\lambda) & =R_{\text {norm }}(\lambda) \times \mathrm{MCHC} \times\left[1-\mathrm{Sat}\left(\mathrm{O}_{2}\right)\right] \times \varepsilon_{\mathrm{Hb}}(\lambda) \times \frac{1}{1-\mathrm{HCT}}, \\
X_{5} & =\frac{\mathrm{HCT}}{\mathrm{MCV}}, \\
X_{6} & =\frac{\mathrm{HCT}^{2}}{\mathrm{MCV}}, \\
X_{7} & =\frac{\mathrm{PCT}}{\mathrm{MPV}} \times \frac{1}{1-\mathrm{HCT}},
\end{aligned}
$$

where $X_{1}$ represented the intercept vector of GLM. Normalized diffuse reflectance, described in Sec. 4.4 was used for the term $\left[R_{m}(\lambda)-c_{1}\right]$ in Eq. (9). Absorption of oxyhemoglobin and deoxyhemoglobin was separated into two predictors, $X_{3}$ and $X_{4} . \mu_{\mathrm{s}, \mathrm{RBC}}^{\prime}$ in Eq. (9) was replaced according to Eq. (6). The HCT-dependent empirical factor, $\gamma(\mathrm{HCT})$, mentioned in Sec. 2.2 , was assumed to be $(1-\mathrm{HCT})^{2}$ or $(1-\mathrm{HCT})(1.4$ $\mathrm{HCT})$. The polynomial terms of $\mu_{\mathrm{s}, \mathrm{RBC}}^{\prime}$ were separated into two predictors $X_{5}$ and $X_{6} . X_{7}$ was used as the replacement of $\mu_{\mathrm{s}, \mathrm{PLT}}^{\prime}$ in Eq. (9). All the predictors were divided by (1$\mathrm{HCT}$ ), i.e., the concentration of extracellular medium. It was cancelled out with the same factor of $\gamma(\mathrm{HCT})$ for predictors $X_{5}$ and $X_{6}$.

The GLM of each wavelength gave statistically significant regression coefficients, except for a few side wavelengths of the spectrum. The significance of the regression coefficients is shown in Table 2 by their ranges of $p$-values through the wavelengths. The correlation coefficient between the reference and predicted FHB concentration ranged from 0.77 to 0.83 for the GLM of each wavelength. The goodness of fits of the models was in a similar range $\left(0.66<R^{2}<0.70\right)$, and the RMS error was around 2.52 to $2.65 \mathrm{~g} / \mathrm{L}$. It was observed that no particular wavelength had a priority for estimating FHB concentration.

Although there was no physical meaning of the regression coefficients individually, some interpretations could be made with their ratios. The ratio of the regression coefficient $b_{5}(\lambda)$ to $b_{6}(\lambda)$ informed us about the $\gamma(\mathrm{HCT})$ because $b_{5}(\lambda)$ and $b_{6}(\lambda)$ are both related to $\mu_{\mathrm{s}, \mathrm{RBC}}^{\prime}$. This ratio was expected to be around -1 or around -1.4 , for consistency with suggested empirical factors in the literature. For most of the spectrum, the ratio was calculated around -1 (mean of wavelengths: -0.9396 , standard deviation 0.0420) [Fig. 11(a)]. Second, the ratio of the regression coefficients $b_{3}(\lambda)$ and $b_{4}(\lambda)$ was expected to be proportional to the ratio of molar extinction coefficients of oxy- and deoxyhemoglobin because they were sample independent factors. The ratio $b_{3}(\lambda) / b_{4}(\lambda)$, plotted in Fig. 11(b), resulted in a good fit with the assumption. In addition, $b_{5}(\lambda) / b_{7}(\lambda)$ or $b_{6}(\lambda) / b_{7}(\lambda)$ should have been consistent with $\sigma_{\mathrm{s}, \mathrm{RBC}}^{\prime}(\lambda) / \sigma_{\mathrm{s}, \mathrm{PLT}}^{\prime}(\lambda)$, which is the ratio of the reduced scattering cross-sections of RBCs and PLTs. According to ratios of these regression coefficients, it was found that $\sigma_{\mathrm{s}, \mathrm{PLT}}^{\prime}>\sigma_{\mathrm{s}, \mathrm{RBC}}^{\prime}$, although the size of PLT is lower than RBC. This result can be explained by the lower anisotropy factor of PLTs compared with RBCs. The ratio of the reduced scattering cross-sections is equal to

$\frac{\sigma_{\mathrm{s}, \mathrm{RBC}}^{\prime}}{\sigma_{\mathrm{s}, \mathrm{PLT}}^{\prime}}=\frac{\sigma_{\mathrm{s}, \mathrm{RBC}}\left(1-g_{\mathrm{RBC}}\right)}{\sigma_{\mathrm{s}, \mathrm{PLT}}\left(1-g_{\mathrm{PLT}}\right)}$,

where $g_{\mathrm{RBC}}$ and $g_{\mathrm{PLT}}$ are anisotropy factors of RBCs and PLTs. The backscattered portion of the scattered light is greater in platelets since $g_{\mathrm{PLT}}$ is lower than $g_{\mathrm{RBC}}$, which was reported in Ref. 28. 


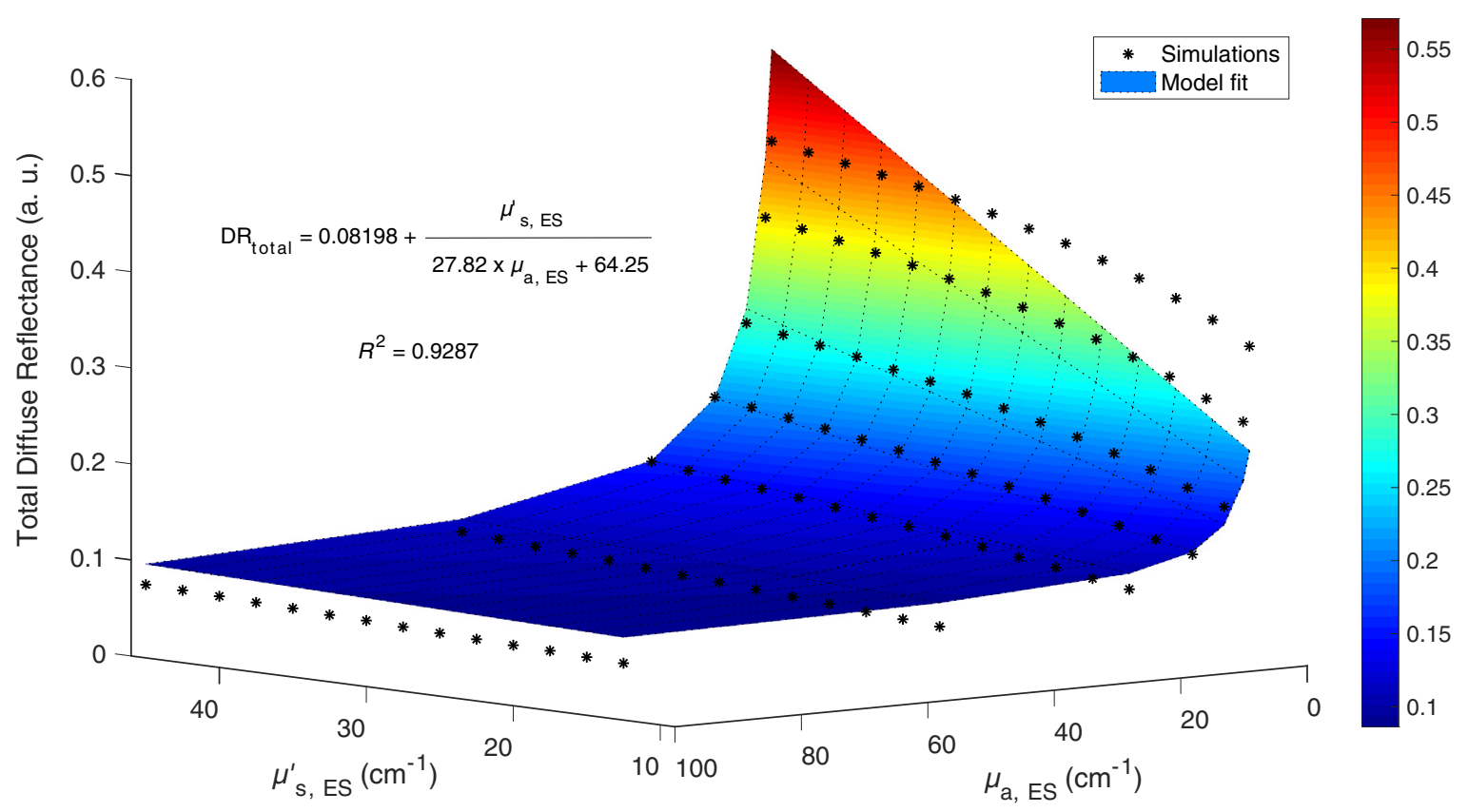

(a)

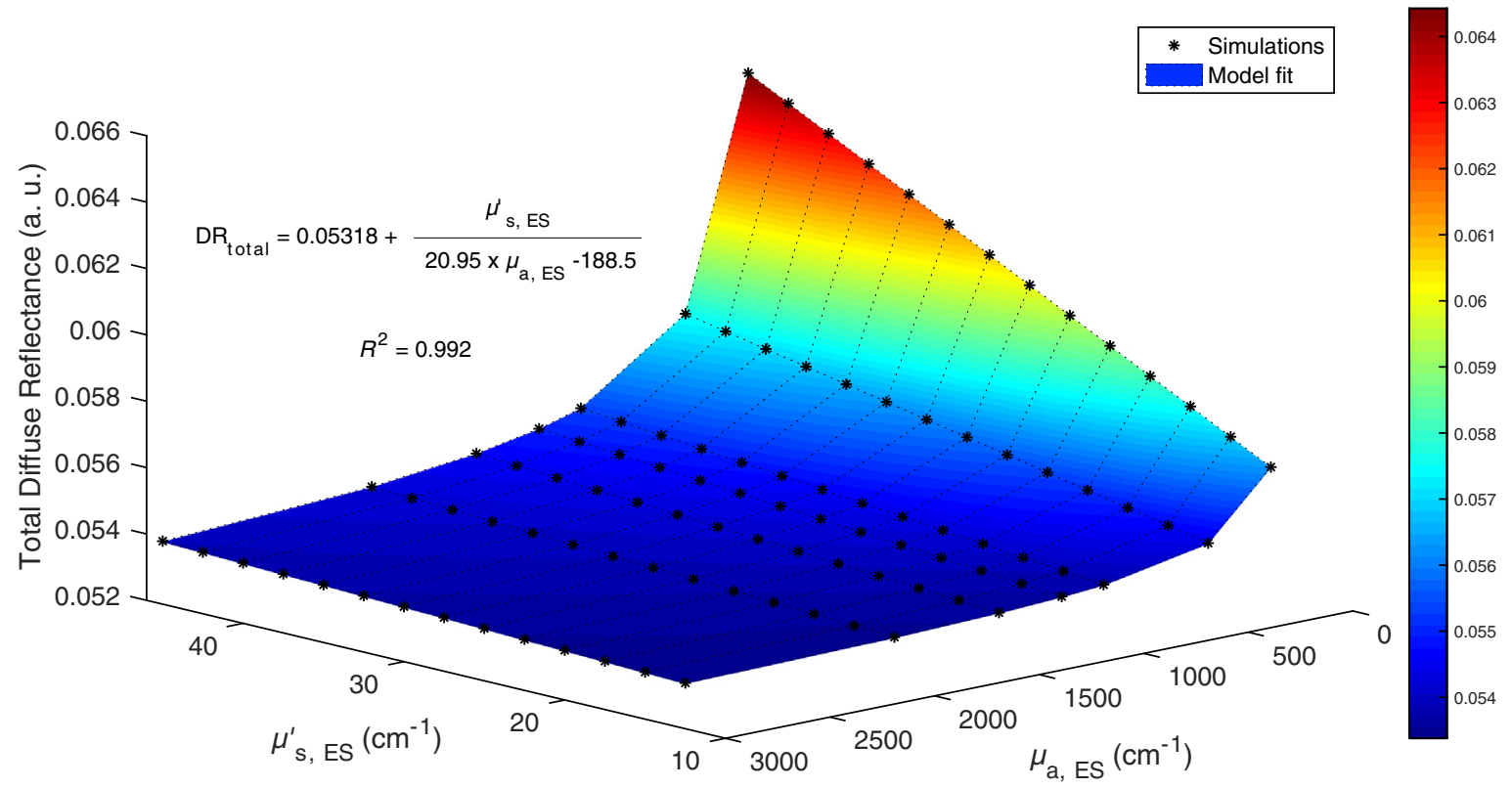

(b)

Fig. 9 MCML simulations: the diffuse reflectance behavior according to the absorption and the reduced scattering coefficients of ES. The model fit to data with (a) low absorption levels and (b) high absorption levels of ES.

\subsection{Accuracy of GLM on Validation Set for Predicting FHB}

The FHB concentrations of the ES units in the validation set were predicted with the regression coefficients computed from the training set. The predictions were compared with the actual FHB concentrations. The optimum results for validation were observed at $568 \mathrm{~nm}$. At this wavelength, the goodness of fit was 0.7999 (Fig. 12) and the correlation coefficient between reference and predicted FHB concentrations was 0.8943. The RMS error was computed as $1.5347 \mathrm{~g} / \mathrm{L}$.

Although predicting the exact value of the FHB could not be achieved with the proposed model, the model predicted and actual FHB concentrations were highly correlated, suggesting that the model could give accurate information about the quality of the blood under storage. The hemolysis levels of ES units were calculated from the following equation after predicting FHB concentrations with the proposed model: 


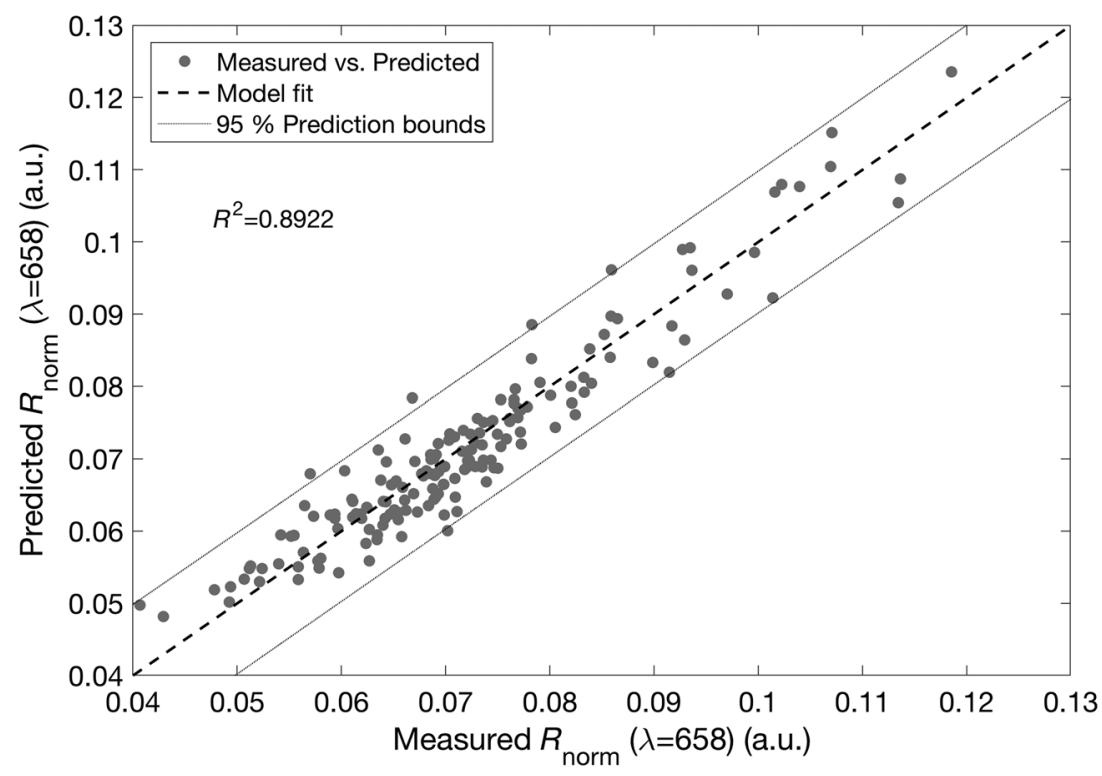

Fig. 10 The fitness of the model on experimental data, at $568 \mathrm{~nm}$, with the highest goodness of fit. One outlier data at $(0.119$ and 0.1513$)$ although included is not visible in the plot range.

Hemolysis level $(\%)=\frac{\text { FHB }}{\mathrm{MCHC}} \times\left(\frac{1-\mathrm{HCT}}{\mathrm{HCT}}\right) \times 100$.

The ES units having hemolysis levels higher than the limit levels were judged as low quality units. The true condition was compared with the assignments according to these predictions. For the threshold of $0.8 \%$ hemolysis, the maximum accuracy was observed as 64/72 ( 89\%) for the model at $568 \mathrm{~nm}$, and, for the threshold of $1.0 \%$ hemolysis, the maximum accuracy was observed as 66/72 ( 92\%) for the model at $562 \mathrm{~nm}$.

\section{Discussion}

In this study, DRS is proposed as a diagnostic tool for the quality assessment of ES units under storage. The model we presented for predicting the FHB concentration has an analytical basis. Predictors include diffuse reflectance measurement of ES units and hematological variables that represent the optical

Table 2 Significance of regression coefficients.

\begin{tabular}{lc} 
Regression coefficients & $p$-value ranges $\forall \lambda$ \\
\hline$b_{1}(\lambda)$ & $4 \mathrm{E}-06$ to 0.013 \\
$b_{2}(\lambda)$ & $1 \mathrm{E}-12$ to 0.058 \\
$b_{3}(\lambda)$ & $1 \mathrm{E}-11$ to 0.096 \\
$b_{4}(\lambda)$ & $1 \mathrm{E}-15$ to 0.002 \\
$b_{5}(\lambda)$ & $9 \mathrm{E}-15$ to $3 \mathrm{E}-05$ \\
$b_{6}(\lambda)$ & $6 \mathrm{E}-17$ to $1 \mathrm{E}-04$ \\
$b_{7}(\lambda)$ & $5 \mathrm{E}-04$ to 0.010 \\
\hline
\end{tabular}
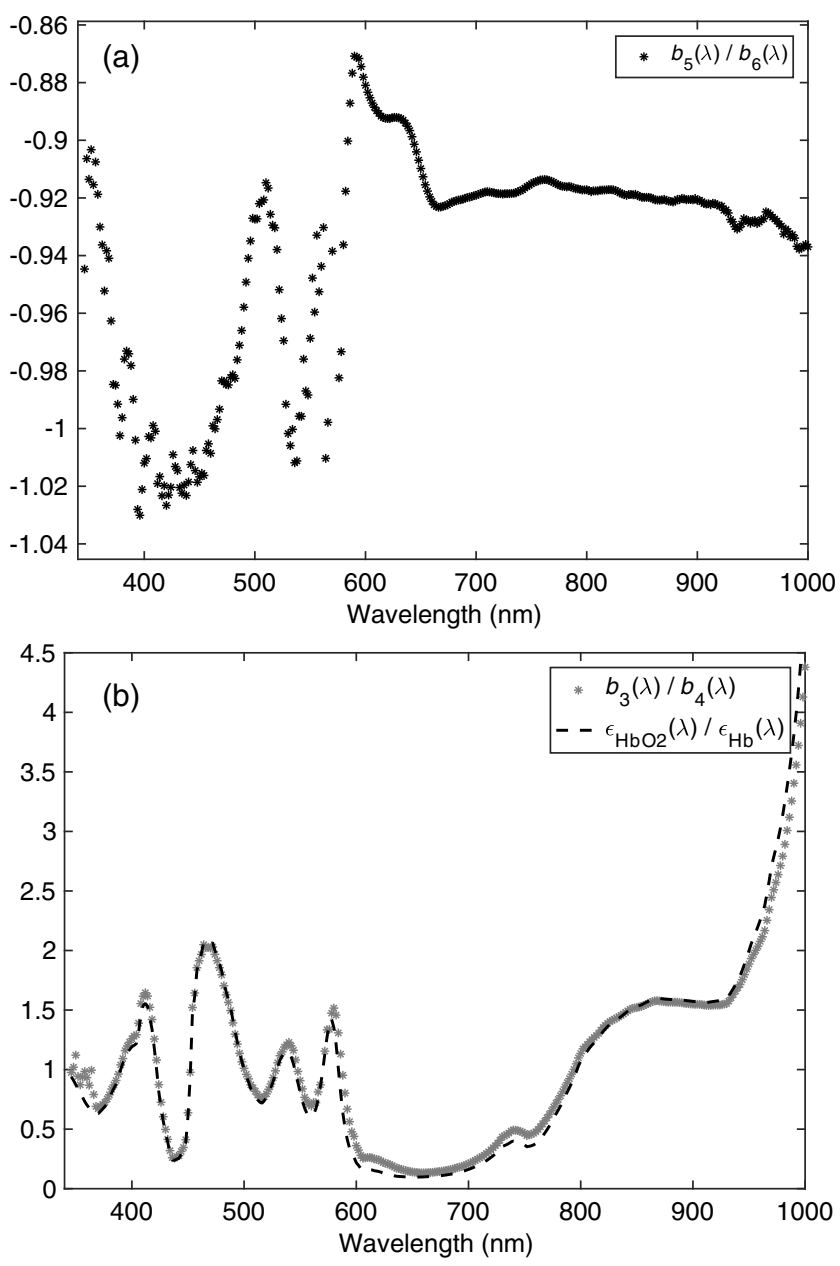

Fig. 11 Ratios of some regression coefficients: (a) $b_{5}(\lambda) / b_{6}(\lambda)$, and (b) $b_{3}(\lambda) / b_{4}(\lambda)$. 


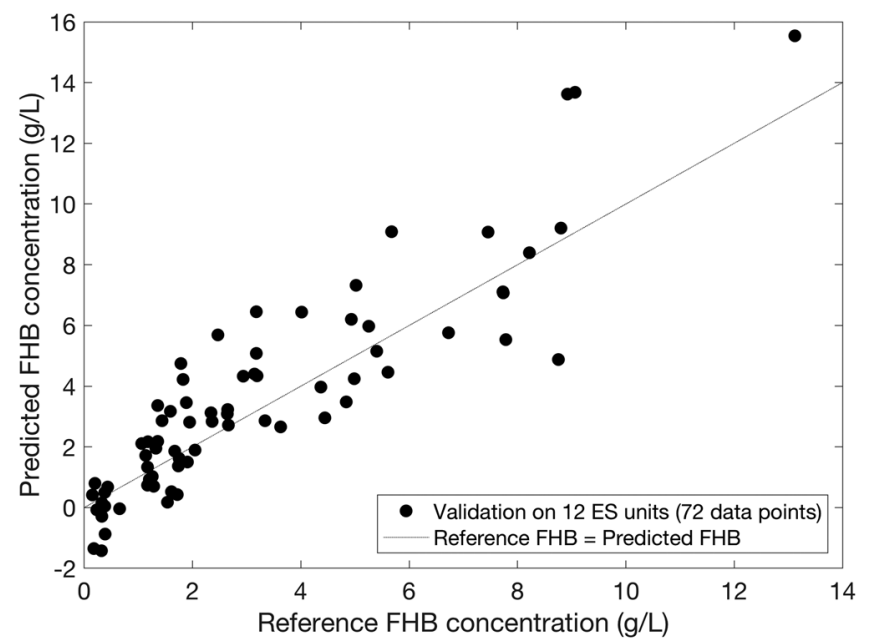

Fig. 12 Estimation accuracy of FHB concentrations of 12 ES units of the validation set, with the model at $568 \mathrm{~nm}$ : reference versus predicted FHB concentrations.

properties of ES. All predictors are statistically significant and excluding any of them would decrease the accuracy of prediction, implying that hematological variables alone could not predict the FHB concentration without diffuse reflectance measurements.

The model is promising, but due to the following reasons there exist some inaccuracies in the results. First, there were numerous particles in the extracellular medium increasing $\mu_{\mathrm{s}, \mathrm{EXT}}^{\prime}$, but, in the modeling, FHB concentration was used as the only source of the increase in $\mu_{\mathrm{s} \text {,EXT. }}^{\prime}$ Second, the optical properties of ES were not physically used; instead, their hematological counterparts were employed. Estimating the optical properties of ES with computational methods such as inverse Monte Carlo would probably provide better results.

Optically measuring the FHB concentration, in the presence of cell interference, has been challenging, and only a few authors have attempted this approach in the past. In one study, the goodness of fit for estimating the hemolysis was evaluated as $R^{2}=0.675$, and the results were improved by fixing the other hematological variables. ${ }^{48}$ In another study, in which transmission spectra were utilized to predict the FHB concentration, the authors reached $R^{2}=0.9524$, but hematological variations in their whole blood samples were not mentioned. ${ }^{49}$ There are multiple factors that affect the optical signal collected from blood samples: aggregation, sedimentation, coagulation, hemolysis, HCT, Sat $\left(\mathrm{O}_{2}\right)$, hemoglobin concentration, etc. Fixing these factors or diluting samples would obviously make the detection of FHB concentrations much easier. In this study, hematological variables were also taken into account and FHB estimation was made on samples with random hematological variables.

In the future, the proposed model can be used for noninvasive estimations of blood quality. Other techniques that noninvasively evaluate the hematological variables of blood should be integrated with DRS. In a recent study, the hemoglobin concentration in stored blood was measured noninvasively with the use of transmission and fluorescence spectroscopy. ${ }^{47}$ Diffuse reflectance measurements by multiple detectors located at various distances from the source gave accurate HCT levels. ${ }^{50}$ The cell counts and volumes could be measured with bioimpedance spectroscopy by designing special blood bags with biocompatible electrodes. ${ }^{51}$

Hemolysis is important in clinical analysis; the FHB content of the blood samples is the largest source of errors in laboratories. Rather than the time-consuming centrifuging and laboratory tests, the proposed model with diffuse reflectance measurements can be integrated into the hematology analyzers.

\section{Conclusion}

In summary, diffuse reflectance spectra of ES units were monitored during storage for estimating the quality of these units, and some remarkable results were observed. Despite significant light absorption and reduced scattering from the blood bag, the diffuse reflectance from the ES units was given as a function of the optical properties of ES. This semiempirical equation was further validated with simulated and experimental data. For modeling the FHB concentration, it was assumed that storage lesions increase the scattering in the extracellular medium. Highlighting the components of the scattering coefficient of ES provided a linear model of FHB concentration with predictors containing hematological variables and diffuse reflectance signal. The presented model gave highly correlated estimates of FHB concentration for each wavelength in the spectrum, but the accuracy of estimating exact values was low. The error rate of the model in diagnosing the true quality was about $10 \%$.

\section{Disclosures}

The authors declare no conflicts of interest.

\section{Acknowledgments}

The study was funded by the Scientific and Technological Research Council of Turkey (TUBITAK) under the grant code 115E477. The authors would like to acknowledge the Directorate General of Blood Services of Turkish Red Crescent for recruitment of samples used in the study and allowing the study to be performed in the Laboratories of Northern Marmara Regional Blood Center, Istanbul. We also thank all the members of Quality Control Laboratory in Northern Marmara Regional Blood Center, Kartal, Istanbul, Turkey, for their assistance and support. The authors would also like to thank Dr. Ercan Kara as the member of Biophotonic Laboratory in Bogazici University for his assistance.

\section{References}

1. K. Pavenski et al., "Red blood cell storage lesions and related transfusion issues: a Canadian blood services research and development symposium," Transfus. Med. Rev. 26(1), 68-84 (2012).

2. S. O. Sowemimo-Coker, "Red blood cell hemolysis during processing," Transfus. Med. Rev. 16(1), 46-60 (2002).

3. J. R. Hess et al., "Red blood cell hemolysis during blood bank storage: using national quality management data to answer basic scientific questions," Transfusion 49(12), 2599-2603 (2009).

4. A. Constituents, P. Function, and S. Life, "1 RBC concentrates," Transfus. Med. Hemother. 36(6), 362-370 (2009).

5. E. Gkoumassi et al., "Hemolysis of red blood cells during processing and storage," Transfusion 52(3), 489-492 (2012).

6. K. A. Janatpour et al., "Visual assessment of hemolysis in red blood cell units and segments can be deceptive," Transfusion 44(7), 984-989 (2004).

7. O. M. Can, Y. Ülgen, and A. Akın, "Use of the color spaces in determining the level of hemolysis in blood under storage," in Proc. of 6th European Conf. of the Int. Federation for Medical and Biological 
Engineering, I. Lackovic and D. Vasic, (Eds.), vol. 45, pp. 290-293 (2015).

8. S. Takatani and M. D. Graham, "Theoretical analysis of diffuse reflectance from a two-layer tissue model," IEEE Trans. Biomed. Eng. BME-26(12), 656-664 (1979).

9. A. Kienle et al., "Noninvasive determination of the optical properties of two-layered turbid media," Appl. Opt. 37(4), 779-791 (1998).

10. D. Yudovsky and L. Pilon, "Simple and accurate expressions for diffuse reflectance of semi-infinite and two-layer absorbing and scattering media," Appl. Opt. 48(35), 6670-6683 (2009).

11. T.-Y. Tseng et al., "Quantification of the optical properties of two-layered turbid media by simultaneously analyzing the spectral and spatial information of steady-state diffuse reflectance spectroscopy," Biomed. Opt. Express 2(4), 901-914 (2011).

12. S. Chandrasekhar, Radiative Transfer, Oxford University Press, London (1960).

13. G. Zonios and A. Dimou, "Modeling diffuse reflectance from semiinfinite turbid media: application to the study of skin optical properties," Opt. Express 14(19), 8661-8674 (2006).

14. G. Zonios and A. Dimou, "Modeling diffuse reflectance from homogeneous semi-infinite turbid media for biological tissue applications: a Monte Carlo study," Biomed. Opt. Express 2(12), 3284-3294 (2011).

15. G. Zonios, I. Bassukas, and A. Dimou, "Comparative evaluation of two simple diffuse reflectance models for biological tissue applications," Appl. Opt. 47(27), 4965-4973 (2008).

16. G. Zonios et al., "Melanin absorption spectroscopy: new method for noninvasive skin investigation and melanoma detection," J. Biomed. Opt. 13(1), 014017 (2008).

17. V. Sharma et al., "Quantification of light reflectance spectroscopy and its application: determination of hemodynamics on the rat spinal cord and brain induced by electrical stimulation," NeuroImage 56(3), 1316-1328 (2011).

18. R. Marchesini, A. Bono, and M. Carrara, "In vivo characterization of melanin in melanocytic lesions: spectroscopic study on 1671 pigmented," J. Biomed. Opt. 14(1), 014027 (2009).

19. J. M. Steinke and A. P. Shepherd, "Diffusion model of the optical absorbance of whole blood," J. Opt. Soc. Am. A 5(6), 813 (1988).

20. A. N. Yaroslavsky et al., "Optical properties of blood in the near-infrared spectral range," Proc. SPIE 2678, 314-324 (1996).

21. D. K. Sardar and L. B. Levy, "Optical properties of whole blood," Lasers Med. Sci. 13, 106-111 (1998).

22. M. Meinke, "Optical properties of platelets and blood plasma and their influence on the optical behavior of whole blood in the visible to near infrared wavelength range," J. Biomed. Opt. 12(1), 014024 (2007).

23. M. Friebel et al., "Determination of optical properties of human blood in the spectral range 250 to $1100 \mathrm{~nm}$ using Monte Carlo simulations with hematocrit-dependent effective scattering phase functions," J. Biomed. Opt. 11(3), 034021 (2006).

24. V. V. Tuchin, Handbook of Optical Biomedical Diagnostics, 2nd ed., pp. 171-216, SPIE Press, Bellingham, Washington (2002).

25. S. L. Jacques, "Optical properties of biological tissues: a review," Phys. Med. Biol. 58(11), R37-R61 (2013).

26. N. Bosschaart et al., "A literature review and novel theoretical approach on the optical properties of whole blood," Lasers Med. Sci. 29(2), 453479 (2014).

27. I. Fredriksson, "Model-based quantitative laser Doppler flowmetry in skin," J. Biomed. Opt. 15(5), 057002 (2010).

28. A. Roggan et al., "Optical properties of circulating human blood in the wavelength range 400-2500 nm," J. Biomed. Opt. 4(1), 36-46 (1999).

29. A. Timothy Lovell et al., "Determination of the transport scattering coefficient of red blood cells," Proc. SPIE 3597 (1999).

30. V. Twersky, "Absorption and multiple scattering by biological suspensions," J. Opt. Soc. Am. 60(8), 1084-1093 (1970).

31. R. J. Zdrojkowski and N. R. Pisharoty, "Optical transmission and reflection by blood," IEEE Trans. Biomed. Eng. BME-17(2), 122-128 (1970).

32. J. M. Steinke and A. P. Shepherd, "Comparison of Mie theory and the light scattering of red blood cells," Appl. Opt. 27(19), 4027-4033 (1988).
33. M. Meinke et al., "Empirical model functions to calculate hematocritdependent optical properties of human blood," Appl. Opt. 46(10), 17421753 (2007).

34. J. R. Hess, "Red cell storage," J. Proteomics 73(3), 368-373 (2010).

35. I. Chin-Yee, N. Arya, and M. S. d'Almeida, "The red cell storage lesion and its implication for transfusion," Transfus. Sci. 18(3), 447-458 (1997).

36. J. Delobel et al., "Red blood cell microvesicles: a storage lesion or a possible salvage mechanism," ISBT Sci. Ser. 11, 171-177 (2016).

37. A. Scott et al., "Determining the optical properties of turbid media by using the adding-doubling method," Appl. Opt. 32, 559-568 (1993).

38. L. Wang, S. L. Jacques, and L. Zheng, "MCML: Monte Carlo modeling of light transport in multi-layered tissues," Comput. Methods Programs Biomed. 47(2), 131-146 (1995).

39. A. A. Stratonnikov and V. B. Loschenov, "Evaluation of blood oxygen saturation in vivo from diffuse reflectance spectra," J. Biomed. Opt. 6(4), 457-467 (2001).

40. H. Chang et al., "Whole blood reflectance for assessment of hematologic condition and detection of angiographic contrast media," Appl. Opt. 48(13), 2435-2443 (2009).

41. E. J. Phelps et al., "Rapid ratiometric determination of hemoglobin concentration using UV-VIS diffuse reflectance at isosbestic wavelengths," Opt. Express 18(18), 18779-92 (2010).

42. A. Sircan-Kucuksayan, M. Uyuklu, and M. Canpolat, "Diffuse reflectance spectroscopy for the measurement of tissue oxygen saturation," Physiol. Meas. 36(12), 2461-2469 (2015).

43. V. Han, K. Serrano, and D. V. Devine, "A comparative study of common techniques used to measure haemolysis in stored red cell concentrates," Vox Sanguinis 98(2), 116-123 (2010).

44. M. Harboe, "A method for determination of hemoglobin in plasma by near-ultraviolet spectrophotometry," Scand. J. Clin. Lab. Invest. 11(1), 66-70 (1959).

45. S. Prahl, "Hemoglobin Spectra," 1999, https://omlc.org/spectra/ hemoglobin/summary.html.

46. M. Meinke et al., "A novel device for the non-invasive measurement of free hemoglobin in blood bags/Messgerät zur zerstörungsfreien Messung von freiem Hämoglobin in Blutkonserven," Biomed. Tech./ Biomed. Eng. 50, 2-7 (2005).

47. S. Zhang et al., "Nondestructive measurement of hemoglobin in blood bags based on multi-pathlength VIS-NIR spectroscopy,” Sci. Rep. 8(1), 1-9 (2018).

48. M. Meinke et al., "chemometric determination of blood parameters using visible-near-infrared spectra," Appl. Spectrosc. 59(6), 826-835 (2005).

49. L. Lin et al., "Detection of free hemoglobin in blood products using transmission spectra and fluorescence spectra for quality assurance," Anal. Methods 8(21), 4239-4244 (2016).

50. J. M. Steinke and A. P. Shepherd, "Reflectance measurements oxyhemoglobin saturation of hematocrit," Am. J. Physiol. 253, C147C150 (1987).

51. O. M. Can, Y. Ülgen, and H. Bilgen, "Monitoring Cole parameters of red blood cell concentrates during storage," in Int. Workshop on Impedance Spectroscopy: Abstract Book, Chemnitz University of Technology, Chemnitz, Germany, p. 96 (2013).

Osman Melih Can received his BS degree in biomedical engineering and minor degree in electrical and electronics engineering from Yeditepe University. He received his MSc degree in biomedical engineering from Bogazici University. $\mathrm{He}$ is $\mathrm{PhD}$ candidate and research assistant at the Institute of Biomedical Engineering in Bogazici University, Istanbul. His research interests focus on diffuse optical spectroscopy and its applications in transfusion medicine. He is a member of SPIE.

Yekta Ülgen is a professor and chair of the Department of Biomedical Engineering at Bahcesehir University, Istanbul. He received his MSc degree and $\mathrm{PhD}$ from Salford University, United Kingdom. His research interests are clinical engineering, bioimpedance measurements, and biomedical instrumentation. 